\title{
Des covariations interfluviales
}

\section{Interfluvial covariations}

\author{
PAR A. COUTAGNE \\ INGENIEUH-CONSLI \\ Ancien Eleve DE L'Ecore Polytechnigue
}

English synopsis p. 218

\section{RESUMẼ DE LA COMMUNICATION}

Deux phénomènes — sans que l'un soit effectivement plus ou moins conditionné par l'autre - peuvent être en corrélation s'ils sont conditionnés l'un et l'autre simultanément par un troisième phénomène ou un certain ensemble de phénomènes. Nous appelons une telle corrélation indirecte covariation : les deux phénomènes ont le même développement historique, et le rapport de corrélation classique $R$, auquel convient mieux le nom de rapport de covariation, chiffre leur synchronisme, leur parallélisme, ou encore le coefficient $r$, employé par nous dans des études analogues, que nous désignons ici par degré de covariation.

\section{Notatrons ET Formules}

Pour le rappel des formules et des définitions, nous renvoyons le lecteur à notre étude « Météorologie et hydrologie », publiée dans la Houille Blanche ( $\mathrm{n}^{\circ} 3$, mai-juin 1948, I. - Considérations préliminaires, pages 229,230 et 231). Ces considérations, relatives aux corrélations pluviofluviales, s'appliquent aux covariations interfluviales par un simple changement de symboles : $Q$ devient $Y$, débit d'un bassin; $H$ devient $X$, débit de l'autre bassin, et l'on obtient les mêmes genres d'expressions et de relations :
Formules de probabilité intrinsèque du débit d'un cours d'eau rapporté à sa moyenne:

$$
\frac{\mathrm{X}}{\mathrm{X}_{0}}=1+\frac{z}{\mathrm{~K}_{1}} \quad \text { et } \frac{\mathrm{Y}}{\mathrm{Y}_{0}}=1+\frac{z}{\mathrm{~K}_{2}}
$$

Formule de covariation théorique correspondante:

$$
\frac{\mathrm{Y}}{\mathrm{Y}_{0}}-1=\frac{\mathrm{K}_{1}}{\mathrm{~K}_{2}}\left(\frac{\mathrm{X}}{\mathrm{X}_{0}}-1\right)
$$

Rapport de covariation :

$$
\mathrm{R}=\frac{\Sigma x y}{\sqrt{\Sigma x^{2} \Sigma y^{2}}}
$$

Formule de covariation associée à une formule de probabilité :

$$
\frac{\mathrm{Y}}{\mathrm{Y}_{0}}-\cdots\left(\frac{\mathrm{X}}{\mathrm{X}_{0}}-1\right)+\frac{z}{\mathrm{~K}}
$$


Degré de covariation :

$$
r=1-\frac{\Sigma|y-\lambda x|}{\Sigma|y|}
$$

Dans la pratique, si le coefficient $\lambda$ est voisin de 1 , on pourra se contenter d'admettre la proportionnalité des débits; on aura les formules simplifiées :

$$
\mathrm{Y}=\frac{\mathrm{Y}_{0}}{\mathrm{X}_{0}} \cdot \mathrm{X}
$$

et :

$$
\frac{\mathrm{Y}}{\mathrm{Y}_{0}}=\frac{\mathrm{X}}{\mathrm{X}_{0}}+\frac{z}{\mathrm{~K}}
$$

\section{Applications}

L'étude de ces covariations interfluviales présente souvent un réel intérêt. Comme applications nous citerons les suivantes :

$1^{\circ} \mathrm{Si}$ la covariation est positive $(\mathrm{R}>0)$ et forte, on peut l'utiliser pour déterminer le régime d'un cours d'eau pour lequel on ne possède des observations directes que pour peu d'années, en fonction des caractéristiques fluviales d'un autre cours d'eau, dont le régime est connu d'après des observations de longue durée, pourvu qu'on ait observé les débits des deux cours d'eau pendant une certaine période commune.

$2^{\circ}$ Les covariations positives et faibles, et à fortiori les covariations négatives conduisent à des enseignements d'un autre ordre. Plus deux cours d'eau sont saisonnièrement ou périodiquement différents, plus il y a intérêt à les conjuguer en vue de leur utilisation commune, de manière à obtenir un ensemble, par exemple hydroélectrique, dont la productivité soit la plus constante possible, soit au cours des différents mois, soit au cours des années successives.

$3^{\circ}$ Une autre application des covariations interfluviales réside dans le fait qu'clles permettent de contrôler, les unes par les autres, les statistiques fluviales, de les corriger, de les compléter. Mais nous devons noter à ce sujet qu'il est des cas où l'on se trouve embarrassé pour dire si un manque - ou une faiblesse de corrélation - est dù à une erreur statistique ou à la réalité des faits. D'autres considérations, par exemple pluvis ou thermofluviales, peuvent entrer en ligne de compte pour trancher la question.

$4^{\circ}$ Si deux bassins sont en forte covariation fluviale, les prévisions de débit faites pour l'un d'eux sont plus ou moins valables pour l'autre: ce qui peut entraîner une simplification des moyens de prévision mis en œuvre et réaliser des économies d'exploitation.

\section{ClassificatTon}

Nous distinguerons, plus particulièrement, dans cette étude, les covariations suivantes :

a) Les covariations périodiques par exemple annuelles. On étudie comparativement les débits annuels successifs de deux bassins;

b) Les covariations mensuelles particulièes, c'est-à-dire correspondant à un mois particulier, ou éventuellement à une saison de plusieurs mois successifs;

c) Les covariations mensuelles générales : tous les mois sont considérés simultanément et indistinctement;

d) Les covariations mensuelles moyjennes tantôt positives, tantôt négatives - résultant de la considératioin de l'année fluviale moyenne, c'est-à-dire des 12 coefficients mensuels de débit de l'un et l'autre des deux bassins étudiés.

Les covariations mensuelles, particulières ou générales ( $b$ et $c$ ), ne sont à envisager que s'il s'agit de deux bassins entre lesquels existe une covariation appréciable des débits annuels.

Par contre, l'étude des covariations mensuelles moyennes (d) n'est pas soumise à cette condition, comme nous le verrons plus loin.

\section{ExEMples}

Nous résumons et commentons brièvement, dans les tableaux ci-après, nos études de covariations interfluviales annuelles et mensuelles particulières (paragraphes $a$ et $b$ ci-dessus). Pour mieux caractériser les débits, nous avons remplacé dans les figures les symboles généraux $\mathbf{Y}$ et $\mathrm{X}$ par les initiales des cours d'eau ou des stations dont ils représentent les débits (exemple: Truyère : $\mathrm{Y}=\mathrm{T}$; Bés : $\mathrm{X}=\mathrm{B}$, etc.).

Le tableau de la figure 1 donne pour quelques couples de cours d'eau les probabilités intrinsèques (formule 1), les covariations approchées associées aux probabilités suivant formule (5) et, éventuellement, les proportionnalités de débits suivant formules $\left(2^{\prime}\right)$ ou $\left(5^{\prime}\right)$.

Le tableau de la figure 2 donne un plus grand nombre de covariations, mais seulement sous la forme exprimée par les formules (2) et, éventuellement, $\left(2^{\prime}\right)$.

Nous donnons ci-après quelques exemples de représentations graphiques de covariations : 
Débits moyens en $\mathrm{m}^{3} / \mathrm{s}(1)$

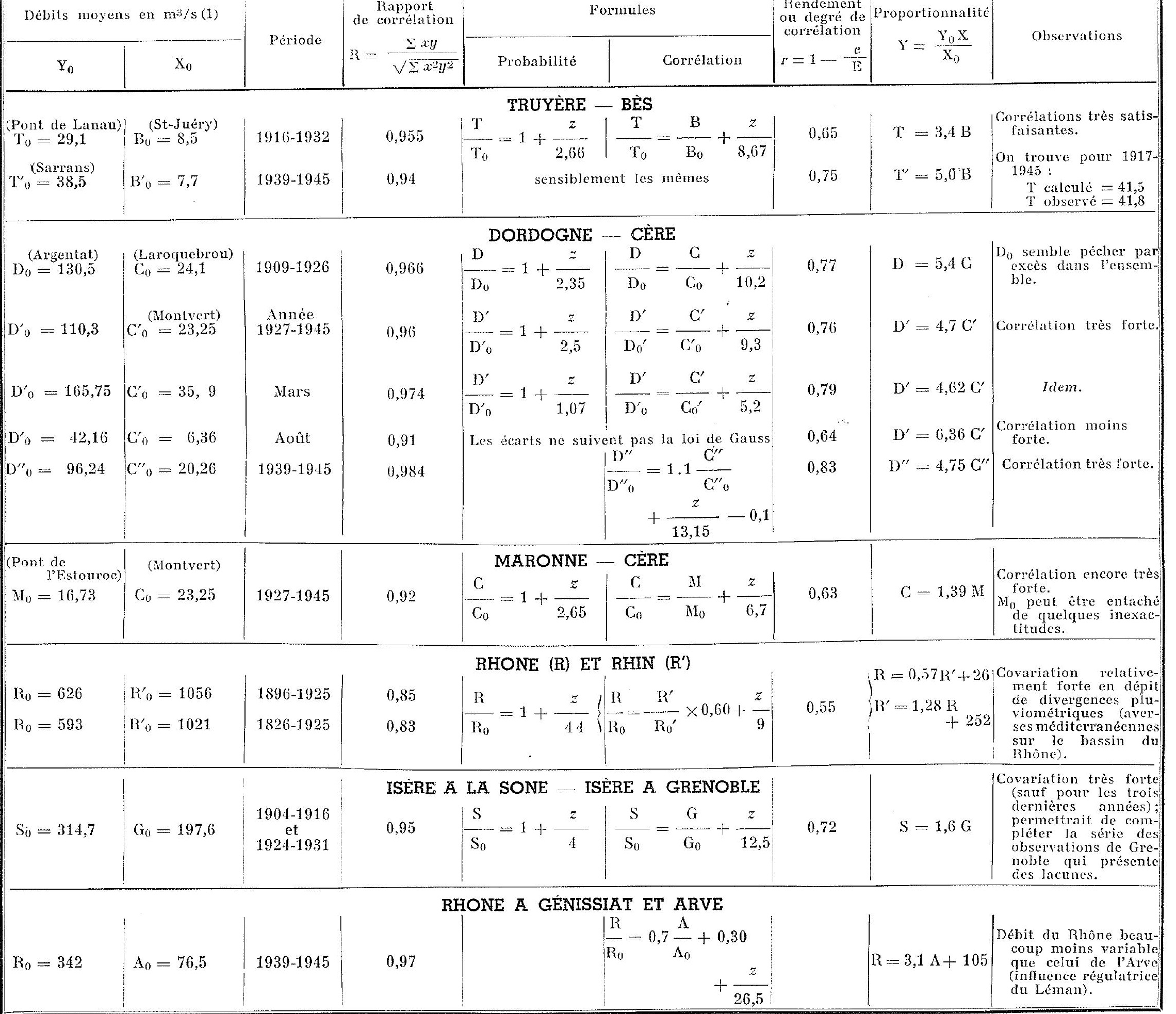


FIG. 2.

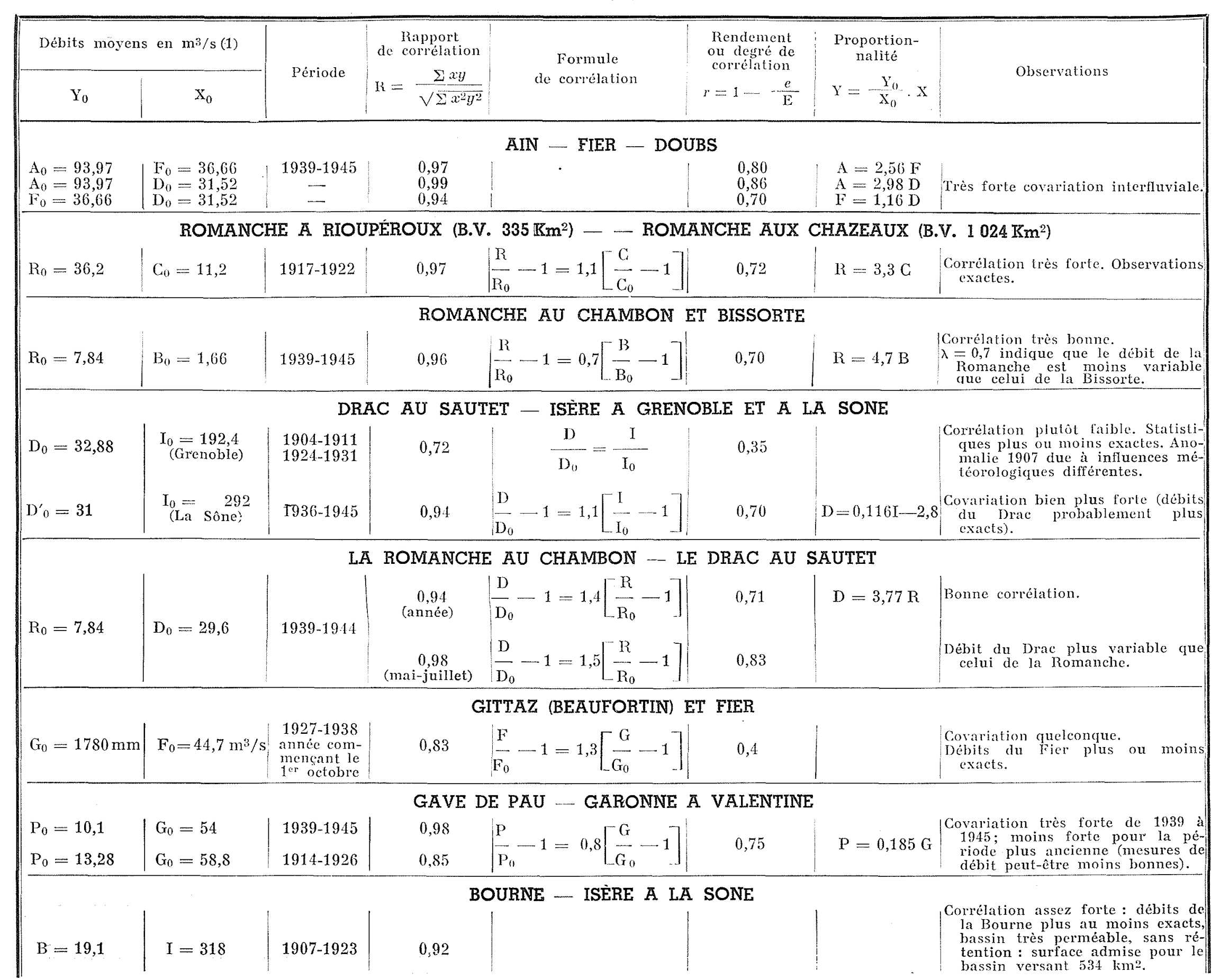




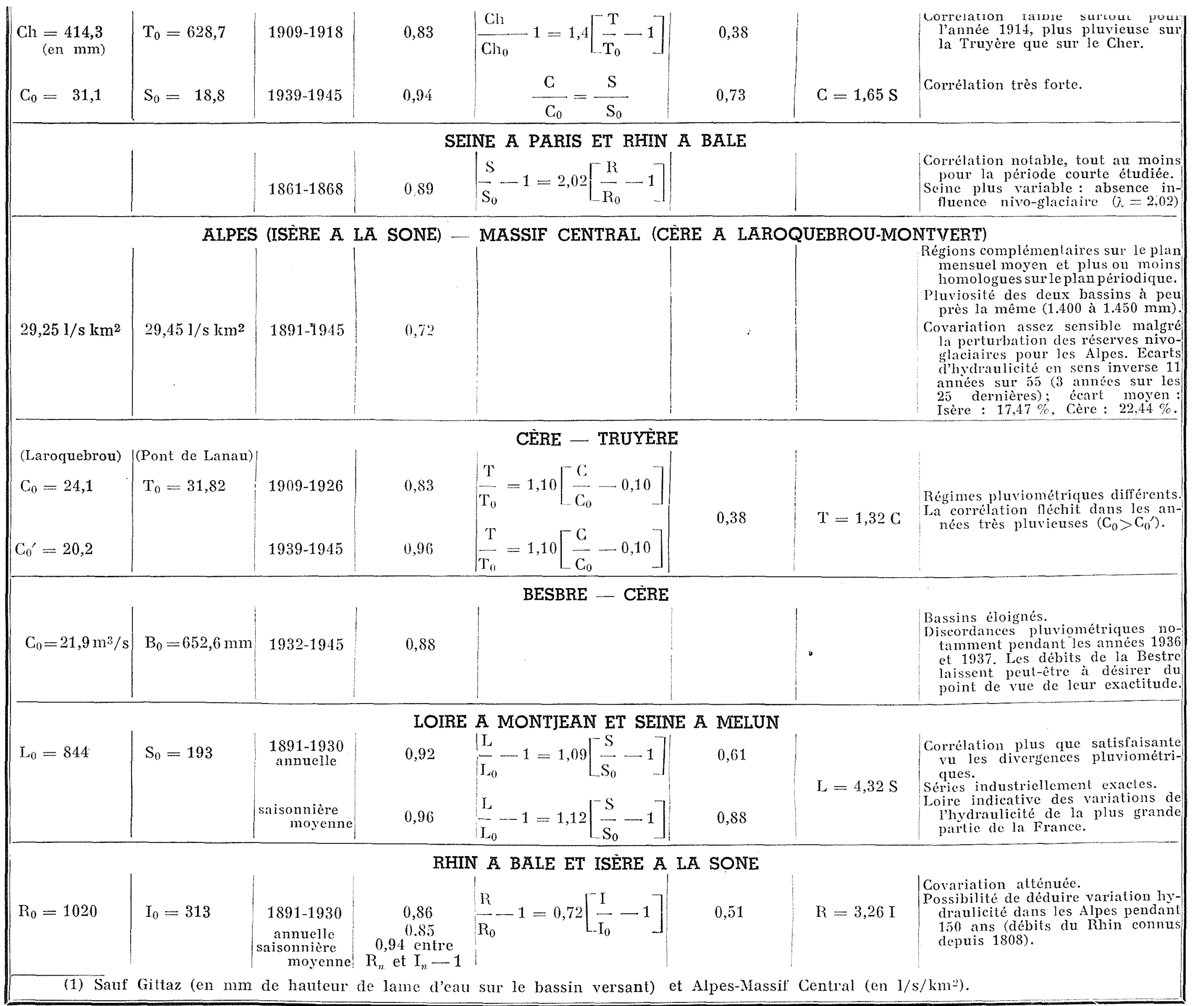




\section{a) Covariations annuelles :}

La figure 3 représente la covariation TruyèreBés suivant la formule (5') durant la période 1916-1932.

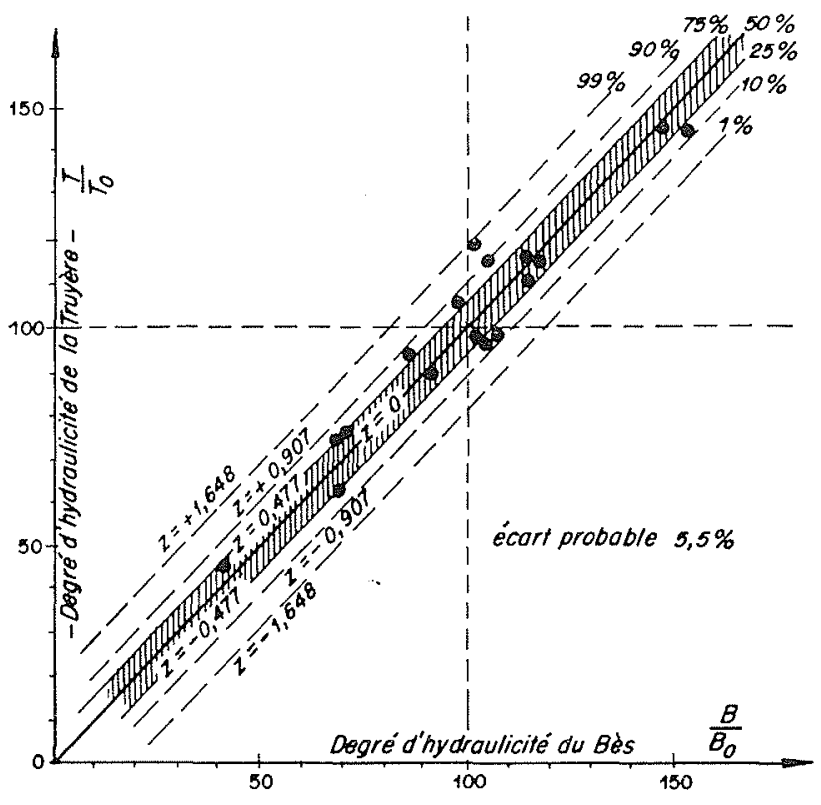

FIG. 3

La figure 4 représente la covariation RhinIsère, par juxtaposition des débits des deux cours d'eau rapportés à leur moyenne respective (hydraulicités annuelles) durant la période 18911930.

La figure 5 donne, pour le Drac au Sautet (1936-1945), un exemple de juxtaposition graphique des débits observés sur cette rivière, et de ses débits calculés par la formule de covariation

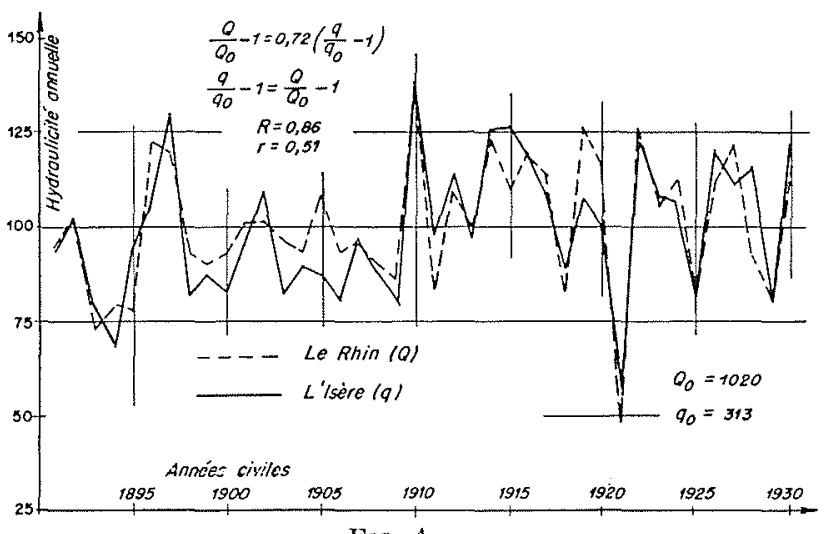

FIG. 4.

(2) en fonction de ceux de l'Isère à la Sône (hydraulicités annuelles).

b) Covariations mensuelles particulieres :

La figure 6 représente, suivant la formule (5'),

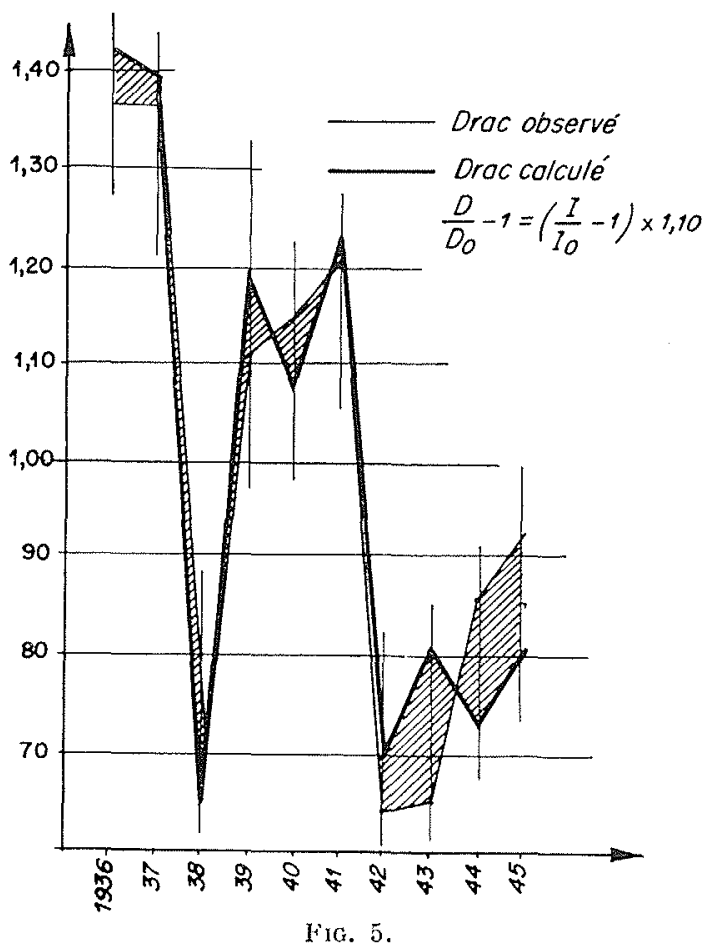

la covariation mensuelle, particulière au mois de mars pour la Dordogne et la Cère dans la période 1927-1945.

c) Covariations mensuelles générales :

Nous avons étudié de ce point de vue :

1) La Truyère et le Bés (1916-1932).

2) La Romanche à Rioupéroux et la Romanche aux Chazeaux (1917-1922).

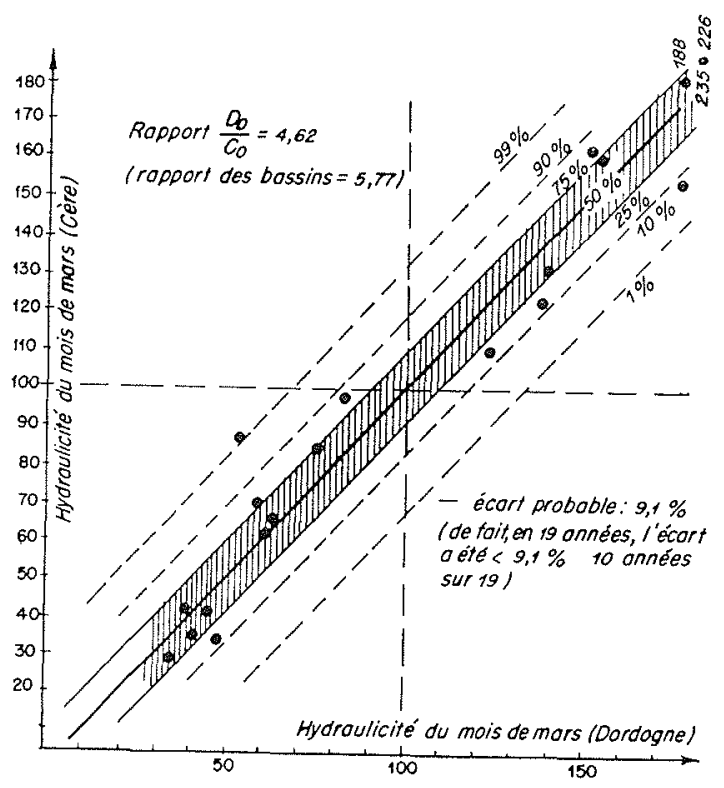

Fig. 6 . 

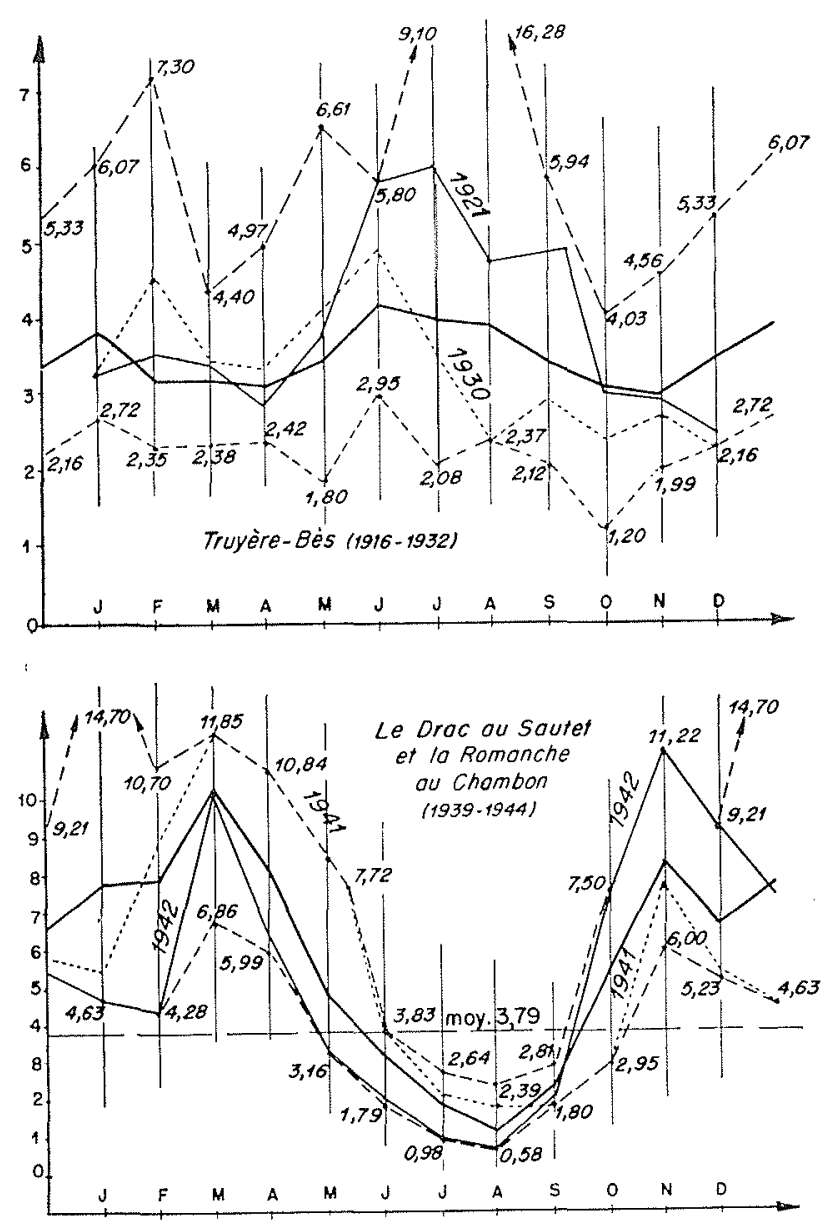

Fig. 7.
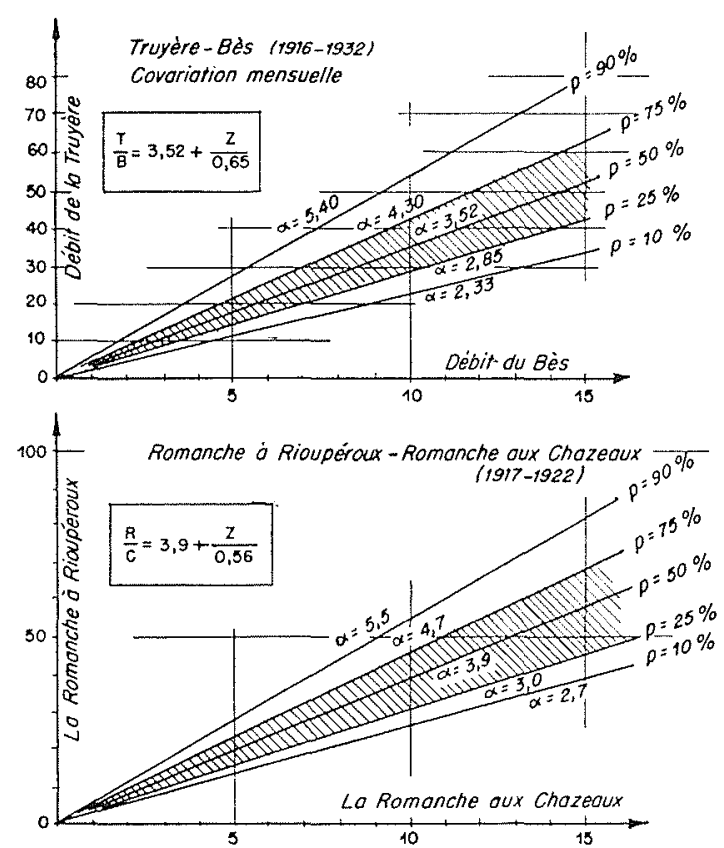

FIG. 8.
3) La Romanche au Chambon et la Bissorte (1939-1944).

4) Le Drac au Sautet et la Romanche au Chambon (1939-1944).

La figure 7 , composée de deux graphiques, représente, pour les couples 1 et 4 ci-dessus, la variation $x=f(t)$, au cours des douze mois de l'année, du rapport $\alpha$ des débits mensuels des deux bassins considérés; chaque graphique comprend les courbes moyennes, les courbes extrêmes et les courbes relatives à deux années particulières.

On peut également, comme mentionné, illustrer la covariation des débits $Q_{1}$ et $Q_{2}$ des deux bassins, par un abaque :

$$
\frac{\mathrm{Q}_{1}}{\mathrm{Q}_{2}}=\alpha+\frac{z}{\beta}
$$

ou, d'une manière plus générale :

$$
\mathrm{Q}_{1}=f\left(\mathrm{Q}_{2}, p\right)
$$

p représentant la probabilité.

La figure 8 donne ces abaques pour les deux premiers couples ci-dessus.

Ceci admet que la variable $\frac{Q_{1}}{Q_{2}}$ suit la loi des erreurs fortuites, ce qui n'est, semble-t-il, pas le cas.

\section{d) Covariations mensuelles moyennes :}

De telles covariations peuvent être étudiées mème sur deux bassins ayant entre eux une covariation annuelle faible ou inexistante, positive ou négative.

Le diagramme de la Romanche (fig. 9), dénote une covariation moyenne très forte (positive)

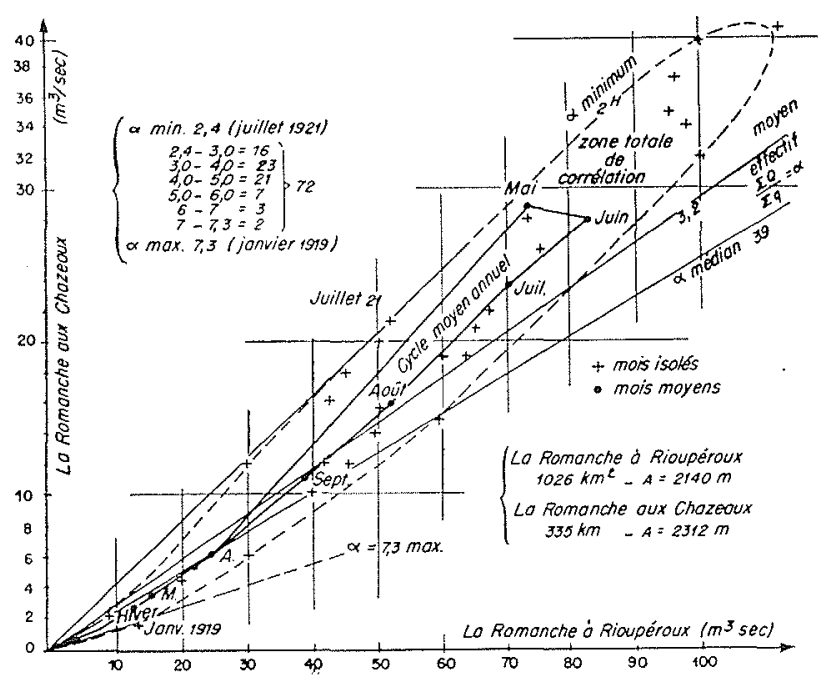

Fid. 9 . 
par la forme allongée du cycle moyen annuel $Q_{1}=f\left(Q_{2}, t\right)$ et de la zone de corrélation totale.

La covariation sera par contre négative entre deux bassins, l'un à alimentation pluviale, l'autre à alimentation nivale ou nivoglaciaire.

C'est grâce à de telles covariations négatives existant entre les rivières des Alpes et celles du Massif Central, par exemple, que la conjugaison des aménagements de ces deux régions permet de réaliser un ensemble saisonnier, en moyenne plus ou moins régularisé. Mais il est inutile d'insister sur ce point trop connu.

Comme exemple, citons la covariation saisonnière moyenne entre la Saône à Lyon et la Romanche à Kioupéroux, que caractérise la valeur $R=-0,73$ du coefficient de covariation.

De telles covariations sont d'ailleurs presque indépendantes des caractéristiques pluviométriques. C'est l'altitude et la température, c'est-àdire la nivosité et la glaciation qui en déterminent la nature, positive ou négative. e) Covariations saisonnières moyennes de la Seine à Ml. lun et de la Loire à Montjean :

La figure 10 donne une idée de cette covariation très satisfaisante mentionnée dans le ta-

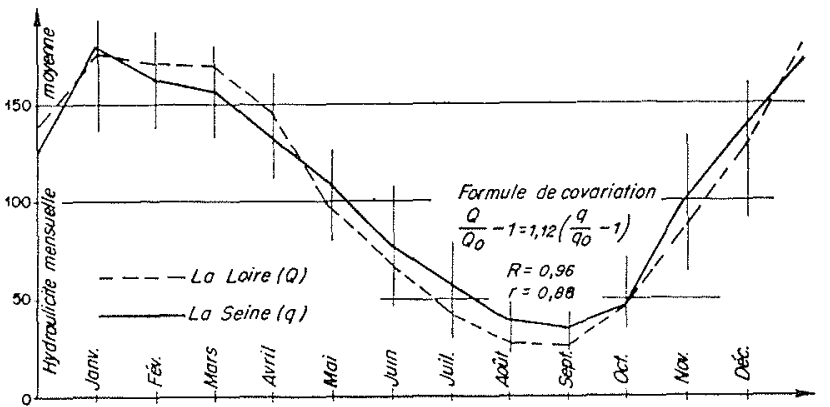

bleau de la figure 2 ; les débits de la Loire sont extraits de l'étude de MM. Pardé et GIBErt : Régime du cours inférieur de la Loire (1).

(1) Voir pages 287 et suivantes du présent numéro.

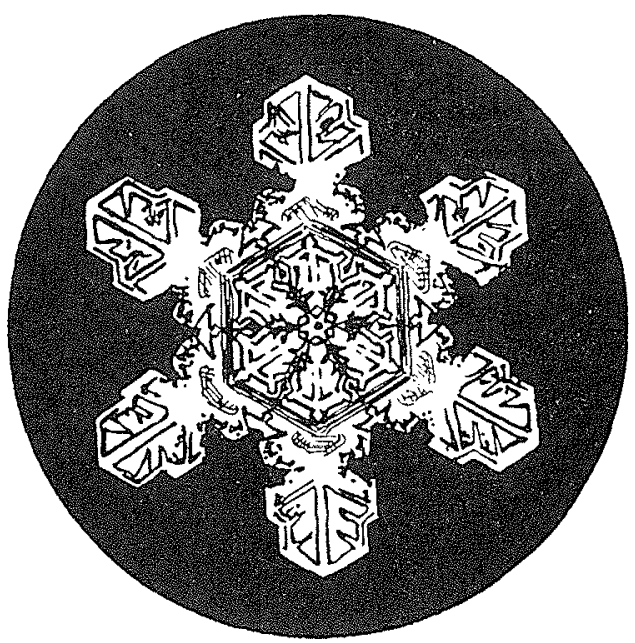

